De la Torre Oliver, Paco.

Profesor, Universidad Politécnica de Valencia, Pintura, CIAE.

\title{
A10tv. Diez años del canal de arte independiente español
}

\author{
TIPO DE TRABAJO:
}

Comunicación.

PALABRAS CLAVE:

Canal de vídeo, Patner YouTube, relato arte contemporáneo, entrevistas, diffusion.

KEY WORDS:

Video channel, YouTube Partner, contemporary art, interviews, broadcast.

\section{RESUMEN}

Presentamos el resultado del trabajo realizado por el canal de vídeo del portal Arte10.com, un relato independiente de la actividad expositiva española en el campo del arte contemporáneo desde el año 2007. Cumpliendo con este objetivo, a lo largo de su trayectoria el canal A10tv ha publicado medio millar de documentos que cuentan con más de quinientas mil descargas. El contenido de su archivo está integrado por registros de exposiciones y actos de inauguración en salas privadas e institucionales, así como entrevistas con artistas españoles contemporáneos.

Su historia corre en paralelo a la de la plataforma Youtube de Google, de cuyo programa partners forma parte desde el año 2010. Esta circunstancia permite ofrecer una lectura desde la experiencia de la distribución de contenidos locales a escala internacional, dentro del llamado fenómeno de la glocalización. Del mismo modo, desde su posicionamiento dentro de las iniciativas pioneras en el campo de la información artística en los inicios de Internet se realiza una reflexión sobre la evolución del sector a lo largo del reciente periodo de crisis y el futuro de los relatos alternativos al discurso único en los medios de comunicación.

\section{ABSTRACT}

We present the result of the work done by the video channel of the site Arte10.com that since 2007 develops an independent account of the spanish exhibition activity in the field of contemporary art. In compliance with this objective, throughout its history the channel A10tv has published half thousand documents with more than five hundred thousand downloads. The content of its archive is integrated by registers of exhibitions and opening acts in private and institutional spaces, as well as interviews with contemporary spanish artists.

Its story runs parallel to that of Google's YouTube platform, of which partners program has been part since 2010. This circumstance allows to offer a reading from the experience of local content distribution on an international scale, within the so-called phenomenon of glocalization. In the same way, from its positioning within the pioneering initiatives in the field of artistic information in the beginnings of the Internet, a reflection is made on the evolution of the sector throughout the recent crisis period and the future of alternative narratives to discourse unique in the media.

\section{CONTENIDO.}

A10tv es un proyecto que nace en el año 2006 en el marco del portal de arte Arte10.com. Desde su concepción, el canal de vídeo apostó por centrar su interés en los artistas y su actividad en la escena española, asumiendo su identidad local en un medio globalizado. La aparición de nuevos medios especializados en arte contemporáneo a través de internet nos animó a poner en marcha un proyecto dirigido por y desde los propios artistas, frente a los portales desarrollados por periodistas y críticos de arte en el seno de 
grupos editoriales. Los contenidos que generaría nuestra actividad tendrían como fin la formulación de respuestas alternativas a las nuevas derivas que la gestión, comercialización y difusión del arte contemporáneo adoptaba en el nuevo siglo.

Los objetivos que nos planteamos al iniciar este proyecto fueron investigar en las relaciones entre los medios de comunicación y el arte contemporáneo en el siglo XXI a través del trabajo de campo, generar documentos audiovisuales en los que ofrecer un relato personal de la actualidad artística contemporánea española, explorar las posibilidades de difusión internacional de contenidos audiovisuales o la decodificación de las claves del control de la información relacionadas con el arte. Y específicamente, con respecto al portal de arte Arte10.com, posicionarlo mediante la incorporación de producciones audiovisuales propias a su oferta de contenidos y estudiar la posible fuente de financiación mediante la monetización de las visualizaciones.

Nuestro campo de investigación se basa en el estudio de las relaciones entre los medios de comunicación y el arte contemporáneo a partir de las iniciativas desarrolladas en el marco de la vanguardia histórica con el objetivo de fundar publicaciones desde donde difundir contenidos programáticos. Citemos las revistas europeas De Stijl (1917-1932), el boletín DADA (1917-1921), Valori Plastici (1918-1922) o Minotauro (1933-1939). Una tradición recuperada a finales de la década de los años 1950 por Guy Devord a través de la revista Internationale situationniste (1958-1972). En el caso español, serán un referente las iniciativas promovidas en el seno del grupo Dau al Set (1948-1956) por Joan Brossa, Arnau Puig, Joan Ponç, Antoni Tàpies o Juan Eduardo Cirlot. Con la llegada de la democracia, en la segunda mitad de la década de los años 1970, se producirá una eclosión en la actividad editorial desde la oficialidad y la marginalidad, como recoge el relato que se presenta en la reciente exposición Gelatina dura1. La puesta en marcha de la feria internacional de arte ARCO y la eclosión de la Movida Madrileña, así como la apertura de centros dedicados al arte contemporáneo en todo el territorio español (MNCARS, IVAM, MACBA,...), potenciará su presencia en los medios generalista siendo el máximo exponente La edad de Oro, programa de TVE presentado y dirigido por Paloma Chamorro en la primera mitad de la década de 1980. En este contexto destacará, por su modo de abordar las publicaciones artísticas desde la visión del creador contemporáneo, la revista Figura (1983-1987) dirigida por los artistas Rafael Agredano, Pepe Espaliú y Guillermo Paneque desde Sevilla.

Para presentar una visión de las revistas de arte españolas vivas, y comprender así el actual estado de la cuestión, revisemos el trabajo publicado por la crítica Elena Vozmediano en el año $2013^{2}$. Una recopilación que abarca desde la clásica Archivos de Arte Valenciano (1915) a las publicaciones surgidas a partir de la eclosión de las nuevas tecnologías e internet a final del siglo XX. Vozmediano reconocerá que las revistas de arte son el principal canal investigador y teórico, así como crítico y divulgativo en el terreno artístico y que, atendiendo a la gran cantidad existente en formato impreso o publicadas a través de internet, podríamos cuestionar la percepción respecto a la escasa producción teórica del arte español.

Una misión ejercida y promovida, dejando a un lado las publicaciones académicas, desde iniciativas privadas de acceso gratuito financiadas, en un mercado tan débil como es el español, fundamentalmente a través de la publicidad. Unos ingresos que se vieron incrementados a principios del siglo XXI debido a la necesidad de las instituciones culturales de posicionarse en internet a través de los medios especializados. Un fenómeno que potenció la aparición de portales de arte contemporáneo en España como Arte10.com (1999), Hispanart (2000), Másdearte (2000), Ubicarte (2000), w3art-Salón Kritic (2004) o, posteriormente, Hoyesarte (2008).

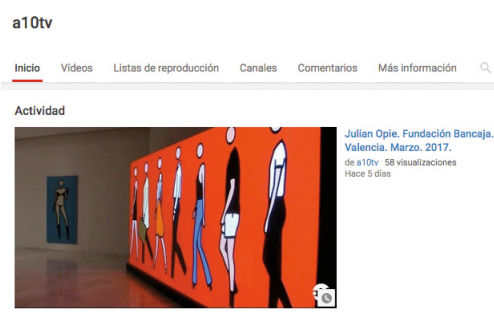

Videos subidos

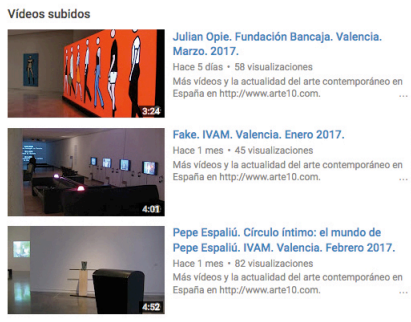

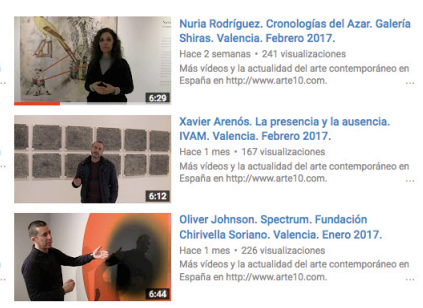

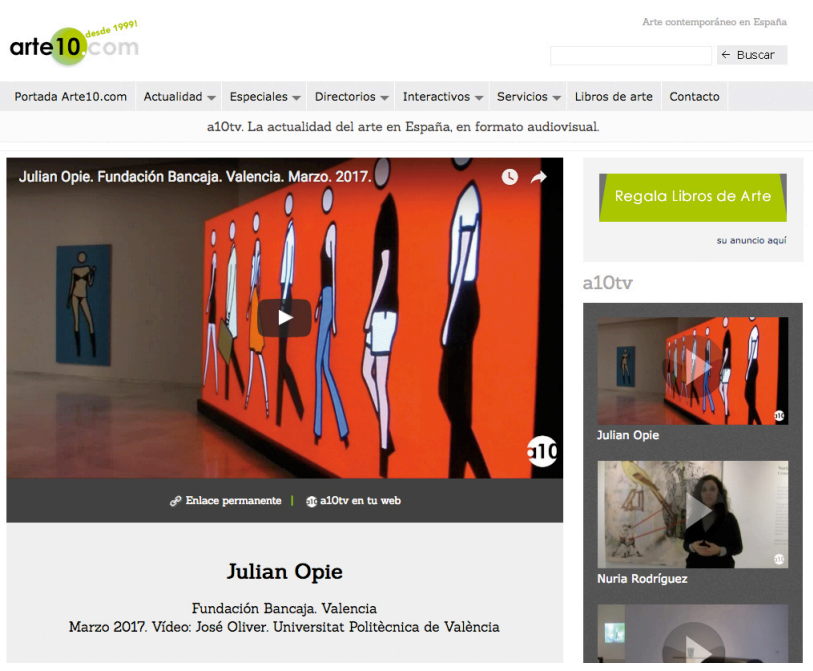

Portadas del canal A10tv en Arte10 y Youtube.

\footnotetext{
${ }^{1}$ Exposición Gelatina dura. Historias escamoteadas de la transición, MACBA, Barcelona, 4/11/2016-19/03/2017.

${ }^{2}$ VOZMEDIANO, Elena. Revistas de arte en España. El Cultural, 21/02/2013. [https://goo.gl/0fjSrT] Consultado el 25/02/2017.
} 
En el año 2000, Arte10.com -en colaboración con Fire Drill ediciones- comenzó a publicar Mundos, una revista on line de carácter experimental donde explorar las posibilidad de la interactividad en la presentación y difusión del arte contemporáneo. Este será el germen de A10tv, un canal de televisión on line desarrollado gracias a la posibilidad de difundir producciones audiovisuales a través de Internet. Desde el inicio del siglo XXI, el vídeo digital se popularizará debido a la comercialización de las cámaras de vídeo digital domésticas y los smartphones, el desarrollo del hardware, la implantación del software de edición digital y la distribución de vídeo streaming a través de internet. La aparición de las redes sociales basada en videos, como Vimeo (2004) o Youtube (2005), posibilitará la transmisión de información desde un nodo emisor a una multitud de nodos receptores de manera simultánea. El Broadcast Yourself eclosionará con la adquisíon de Youtube por Google en el año 2006, convirtiéndose en el referente de vídeo streaming a escala global. Este hecho nos animará a abrir una cuenta gratuita y subir nuestro primer vídeo a finales de ese año.

La posibilidad de distribuir contenidos audiovisuales de producción propia internacionalmente a través de nuestro portal con el soporte de Youtube representó una gran motivación para desarrollar nuestro trabajo a pesar de que nuestro campo de acción se limitaba al ámbito español. En aquel momento, cuando hablábamos de internacionalización y nos enfrentábamos a la dicotomía entre lo local y lo global, no éramos conscientes del efecto de glocalización que sufren estos contenidos al ser difundidos en internet. En el análisis del fenómeno Youtube publicado en 2012 por los investigadores Gallardo y Jorge ${ }^{3}$, determinaron las preferencias por contenidos locales de los internautas españoles dentro de la oferta global de la plataforma Youtube. Durante el periodo de estudio el $77,8 \%$, de los 405 vídeos más vistos en la versión Youtube España ${ }^{4}$, respondían a alguno de los tres rasgos necesarios para ser calificado como español: texto, audio y contenido. Una demostración de que ante la globalidad, dejando a un lado las estrategias del buscador Google, lo local interesa. Gallardo defiende "el triunfo de lo glocal ante lo global" 5 en empresas multinacionales como Youtube, que buscarían la rentabilidad económica con estrategias glocalizadoras a través de herramientas como la geolocalización: "Ios usuarios de Youtube España prefieren los vídeos relacionados con su identidad cultural (local) a pesar de la potencialidad que le da este sitio web de vídeos mundial (global)". Para concluir señalando que: "lo local no solo no está en peligro, sino que puede convertirse en el pilar esencial de los negocios basados en internet". Siguiendo esta lógica no debe extrañarnos que, en junio del año 2007, Youtube tradujera su interfaz a las lenguas locales posicionándose en junio de 2008 con el 38 \% de los vídeos visualizados en Internet. El paso del tiempo parece no haberle restado vigencia al estudio, ya que si revisamos los datos ofrecidos por Youtube en el año 2015, con motivo de su décimo aniversario, el contenido de los 10 vídeos más populares visualizados en España desde su creación son mayoritariamente españoles ${ }^{6}$.

Nuestros principales referentes en este sector emergente fueron dos iniciativas pioneras, una internacional y otra local, cuyo objetivo era poner a disposición de la comunidad artística una serie de servicios dirigidos a la promoción, difusión y producción cultural. VernissageTV (2005) se presentaba como "un proyecto de arte televisivo único de Internet, que abarca exposiciones y eventos en el ámbito del arte contemporáneo, el diseño y la arquitectura" ${ }^{7}$. Nonsite (2003), por su parte, era un proyecto dirigido por los artistas y profesores de la Universitat Politècnica de València, Salomé Cuesta y Bárbaro Miyares, orientado a la producción, difusión y distribución de las prácticas artísticas y visuales contemporáneas. Ambas propuestas destacaban por el tratamiento de los contenidos y la alta definición de la imagen lograda, en gran medida, gracias a la distribución desde su propio servidor o la plaforma Vimeo.

Siguiendo su ejemplo, en el año 2007 experimentamos en ambas prácticas pero las desestimamos debido a las limitaciones en el uso de la alta definición por parte de Vimeo y el alto consumo de ancho de banda del alojamiento de los videos HD. La solución la ofreció de nuevo Youtube, implementando en el año 2008 la posibilidad de alojar videos HD con calidad 720p, y un año más tarde en calidad 1080p. Aunque nuestra permanencia en la plataforma de Google se consolidó el 13 de octubre de 2010 cuando, después de una ardua negociación y gracias a cumplir con los exigentes requisitos de calidad, entramos a formar parte del Programa de Partner de Youtube. A partir de ese momento, como generadores de contenidos oficiales, pudimos monetizar nuestros vídeos, acceder a la herramienta de Sistema de Gestión de Contenido y elaborar estudios de usuarios a través del programa Google Analytics.

Desde nuestro primer vídeo -publicado el 23 de septiembre de 2006 y dedicado a la exposición Los murmullos del artista Chema López celebrada en la galería Tomás March de Valencia- nuestra actividad se orienta a realizar un trabajo de documentación de exposiciones de arte contemporáneo realizados en España. Nuestro archivo esta integrado por reportajes de exposiciones, actos de inauguración y entrevistas con los artistas más representativos del panorama artístico actual.

\footnotetext{
${ }^{3}$ GALLARDO, J. y JORGE, A. (2012). El caso Youtube España: el fenómeno glocal en las redes sociales. Telos, n 92, Julio- Septiembre 2012. [https://goo.gl/BQRdEh] Consultado el 25/02/2017.

${ }^{4}$ Youtube España: lengua española y ubicación España.

${ }^{5}$ GALLARDO CAMACHO, Jorge. Análisis del fenómeno Youtube en España: Relación con los espectadores y con los generadores de contenidos tradicionales. Luciérnaga, Politécnico Colombiano Jaime Isaza Cadavid, 2013, pp. 57- 68.

${ }^{6}$ 1. Pollito Amarillito; 2. Miliki, canciones infantiles, los payasos de la tele; 3. Color Songs Collection Vol. 1; 4. Mundo Pocoyó; 5 . Parodia PSY - Gangnam Style "En el Paro Estoy"; 6. Tomorrowland 2012; 7. La bata manta; 8. Seguiremos; 9. Spot fuet Espetec Casa Tarradellas; 10. La niña repelente. Fuente: Youtube celebra su décimo aniversario en España con sus 10 vídeos más virales. El Periódico, 18-05-2015. [https://goo.gl/Wisqsg] Consultado el 25/02/2017.

[http://vernissage.tv] Consultado el 25/02/2017.
} 
La primera entrevista, publicada el 25 de noviembre de 2006, fue realizada a la artista Mavi Escamilla con motivo de su exposición La vie en rose en la galería Rosa Santos de Valencia. Desde entonces, hemos publicado entrevistas a artistas como Curro González, Alex Francés, Manu Muniategiandikoetxea, Manuel Sáez, Teresa Tomás, Marta Serna, Xisco Mensua, Teresa Moro, Marina Núñez, Carlos Pérez Siquier, Joan Cardells, Txusto Poyo, Ciria, José Manuel Ballester, José Saborit, Enrique Zabala, Andrés Rábago, José Miguel Pereñíquez, Pedro Esteban, Alex Marco, Miki Leal, Rafa Tormo, Darío Villalba, Judas Arrieta, Laboratorio de Luz, Monique Bastiaans, Cabello-Carceller, Javier Garcerá, Yturralde, Arma Álvarez-Laviada, Oliver Johnson o Xavier Arenós, entre otros.

Actualmente contamos con 806 suscriptores y mediante la fuente Analytics conocemos los datos del Lifetime respecto a la actividad de los 575 vídeos disponibles en nuestro canal. Desde el 1 de abril de 2007 al 19 de febrero de 2017 se han realizado un total de 550.913 visionados y los vídeos mas populares son los dedicados a Yoshitomo Nara y Graf con un 4,8\%; Julio González 3,2\%; Fernando Botero 2,9\%; L3C 2,0\%; Cosas del Surrealismo 1,8\%; Tony Ousler 1,7\%; Henri Michaux 1,6\%; Benardí Roig 1,4\%; Figuraciones en La Caixa $1,2 \%$ y Zaj $1,2 \%$.
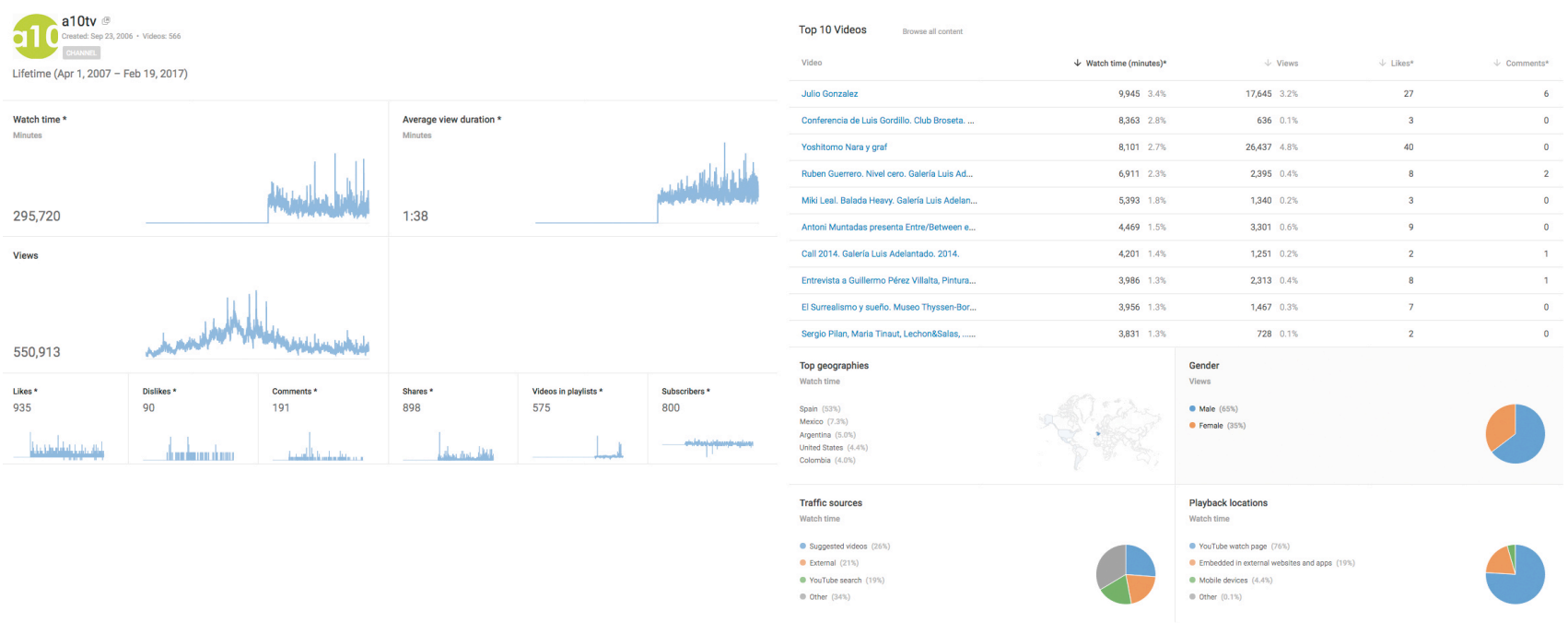

Gráficas y datos del canal a10tv ofrecidos por Google Analytics el 19 de febrero de 2017.

Las visitas proceden de 190 países distribuidos en un $65 \%$ de público masculino y un $35 \%$ femenino. España se encuentra a la cabeza con el $38 \%$, menos de la mitad de las descargas. En la lista de los veinticinco primeros puestos le sigue Mexico con el 8,5\%, Estados Unidos 6,8\%; Argentina 5,1\%; región desconocida 5\%; Colombia 4,7\%; Japón 2,9\%; Italia 2,1\%; Francia 2,1\%; Chile 2,1 \%; Peru 2,1\%; Brasil 2,1\%; Alemania 1,7\%; Reino Unido 1,3\%; Venezuela 1,3\%; Portugal 0,9\%; Holanda 0,8\%; Canada 0,8\%; Ecuador 0,8\%; Bélgica $0,8 \%$; Russia $0,7 \%$; Taiwan $0,6 \%$; Uruguay $0,6 \%$; Hungría $0,4 \%$ y Suiza $0,4 \%$.

Sin embargo si, como hemos señalado, en sus inicios Arte10.com contó con el apoyo de instituciones, fundaciones y entidades privadas, a lo largo de esta última década se ha producido un cambio de tendencia en el mercado publicitario que ha influido determinantemente en el sector editorial. La aplicación de los recortes presupuestarios, motivados por el inicio de la crisis económica internacional en el año 2007, provocará un ciclo bajista en España que se agravará debido a una recesión irreversible como consecuencias del rescate financiero del año 2012 y su repercusión en las grandes cajas española y sus fundaciones, verdaderos motores de la actividad económica en el sector artístico español. El director del Museo Nacional Centro de Arte Reina Sofía, Manuel Borja-Villel, señalaba en el año 2013 que el museo había tocado fondo tras un recorte presupuestario del 25,5\%, a pesar de reducir gastos estructurales e incrementar los ingresos propios ${ }^{8}$. Desde esa inflexión se ha desarrollado un largo proceso que ha provoca la paulatina desaparición de la gran mayoría de portales, y ha debilitado a las publicaciones del sector especializado en arte contemporáneo.

\footnotetext{
${ }^{8}$ Redacción. El director del Reina Sofía asegura que el museo ha tocado fondo. Europa Press, 11/09/2014. [https://goo.gl/cmZ6lk] Consultado el 25/02/2017.
} 
Si las revistas académicas ${ }^{9}$ logran mantenerse gracias a su carácter institucional, publicaciones independientes emblemáticas como Lápiz (1982), Exit (2000), Brumaria (2002) o Dardo (2006), se refugian en la resistencia mientras que otras claudican. El crítico Fernando Huici, director de Arte y Parte, anunciaba a finales del año 2016 el cierre de la revista, después de 125 números publicados a lo largo de lo últimos 20 años. El director de Arte10.com, Jorge Tarazona, publicaba un post al respecto en la página oficial del canal en Facebook donde reaccionaba ante este desenlace cuestionando la supervivencia de las revistas de arte en España, y si tal hecho importaría a alguien: "Si nos quedamos sin medios especializados, todo se convertirá en vulgares likes y corazones" -en referencia a la dinámica propia de las redes sociales. En su reflexión, señalaba la responsabilidad de los museos e instituciones en la situación que atravesaba el sector ya que primaban la autoedición frente a la publicidad en detrimento de la crítica independiente. Pero no pensemos que se trata de un fenómeno local, un referente internacional como ha sido la revista Parkett se suma a la lista de víctimas de los nuevos hábitos de los lectores, tras 100 números y 33 años de existencia. Von Graffenried y sus socios Dieter von Graffenried y Jacqueline Burckhardt señalan que, de momento, para su revista no existe un modelo de negocio viable en este mundo digital, al tiempo que cuestionan las posibilidades de Internet como única herramienta para realizar un análisis profundo del arte 10 .

Actualmente en España los museos e instituciones 11 han minimizado o eliminado los presupuestos destinados a campañas publicitarias, mientras que ponen en marcha iniciativas como el portal Promoción del Arte ${ }^{12}$, del Ministerio de Educación, Cultura y Deporte, que el histórico papel de los medios especializados llegan a desdibujar los límites entre la investigación y la propaganda, como en el caso de Oral Memories ${ }^{13}$. Otro ejemplo de esta tendencia sería el aumento de publicaciones vinculadas a los museos como Radar (MUSAC) o CARTA (MNCARS). Y, podría ser excepcional el caso de Cuadernos del IVAM (2004-2012) -donde durante años han colaborado profesionales vinculados a diversos suplementos culturales de medios generalistas españoles-, pero la iniciativa del departamento de publicaciones del IVAM -valorado en 2,4 millones de euros- está siendo objeto de investigación por posibles irregularidades en su gestión ${ }^{14}$.

En la editorial del primer número de la revista CARTA su director, Manuel Borja-Villel, reflexionaba sobre el papel de la publicación institucional, al que define no como un mero portavoz de las propuestas que tienen lugar dentro del museo sino como "parte integral del mismo proyecto de interpelación social, que no puede prescindir del potencial discursivo y crítico del medio impreso" 15 . Destacando su papel dentro del dispositivo crítico y autocrítico que pretende ser el museo, cuya lectura debería suscitar una inquietud ante la naturaleza de la cultura contemporánea, en lugar de reconducirla hacia el ocio desde las redes de la sociedad del espectáculo.

¿Pero no correrían las instituciones con estas actitudes el peligro de caer en la endogamia y acabar aislándose de la sociedad a la que se dirigen, propiciando una especie de circuito cerrado que ofrece una versión de los hechos sin la distancia crítica necesaria?

Desde A10tv creemos en la necesidad de elaborar relatos independientes que testimonien la pluralidad en la que vivimos, especialmente en el contexto artístico, y defendemos el papel que deben jugar los medios de comunicación y los artistas en su construcción y difusión. Los retos a los que nos enfrentamos estarán regidos por la necesidad de reformular nuestra financiación dentro de la manera de entender el mundo que impone el nuevo sistema económico imperante. La crisis del sector debido a los problemas derivados de las caída de ventas e ingresos publicitarios, unidos a la tendencia hacia la gratuidad de la información, agravan la situación del débil mercado artístico español. Por lo que sospechamos qué, si no se produce un cambio en la actitud de las instituciones culturales, podríamos estar abocados a una nacionalización encubierta de la información.

\footnotetext{
${ }^{9}$ Entre las revistas académicas españolas podemos destacar: Arte y Políticas de Identidad (2009) de la facultad de Bellas Artes de la universidad de Murcia; Arte y sociedad (2011) de la universidad de Málaga; Arte, Individuo y Sociedad (1988) de la universidad Complutense de Madrid; Fedro (2004) de la universidad de Sevilla o Laocoonte (2014) de la universitat de València.

${ }^{10}$ CARRIZO, Rodrigo. Parkett, la revista referencial del arte, cierra por los hábitos digitales. El País, 12/03/2017. [https://goo.gl/CcAjzR] Consultado el 12/03/2017.

${ }_{11}^{11}$ CDAN, MNCARS, Fundación Joan Miró, IVAM, Centro de Arte Dos de Mayo, EACC, CAAM, MACBA, MUSAC, ...

${ }^{12}$ Subdirección General de Promoción de las Bellas Artes: "Somos una unidad del Ministerio de Educación, Cultura y Deporte. Nos encargamos de promover las artes plásticas y la creación contemporánea mediante el desarrollo de un programa permanente de exposiciones temporales y de actividades en torno a la fotografía, el arte contemporáneo y las artes visuales. Así mismo impulsamos las condiciones que mejoren la formación de sus agentes e impulsen la difusión y el conocimiento de su labor, tanto en el ámbito local, como en el nacional e internacional. Fuente: [http://www.promociondelarte.com] Consultado el 25/02/2017.

${ }^{13}$ Oral Memories es un proyecto gestionado por el Servicio de Comunicación de Promoción del Arte.

14 LAMARCA, Eva. Corrupción en el IVAM: billetes de 500, obras falsas y expos fraudulentas. Vanity Fair, mayo 2016. [https://goo.gl/pX81NZ] Consultado el 25/02/2017. ,

${ }^{15}$ BORJA-VILLEL, Manuel. ¿Pueden los museos ser críticos? Carta, nº1, primavera-verano 2010, pp. 1-2.
} 